TITLE:

\title{
Mechanism of microfibril contraction and anisotropic dimensional changes for cells in wood treated with aqueous $\mathrm{NaOH}$ solution
}

\author{
$\operatorname{AUTHOR}(\mathrm{S})$ : \\ Nakano, Takato
}

\section{CITATION:}

Nakano, Takato. Mechanism of microfibril contraction and anisotropic dimensional changes for cells in wood treated with aqueous $\mathrm{NaOH}$ solution. Cellulose 2010, 17(4): 711719

\section{ISSUE DATE:}

2010-08

URL:

http://hdl.handle.net/2433/126692

\section{RIGHT:}

The original publication is available at www.springerlink.com; この論文 は出版社版でありません。引用の際には出版社版をご確認ご利用くだ さい。; This is not the published version. Please cite only the published version. 

for cells in wood treated with aqueous $\mathrm{NaOH}$ solution

Takato Nakano*

22 Key words: anisotropic dimensional change, microfibril, sodium hydroxide solution, swelling,

23 shrinking, cell wall

24

25 Received $* * * * * *$; accepted $* *, * * * *$

26 * Laboratory of Biomaterials Design, Division of Forest and Biomaterials Science, Graduate

27 School of Agriculture, Kyoto University, Kita-Shirakawa, Kyoto, 606-8502 Japan. 
28 Abstract

Anisotropic swelling of wood samples was observed upon treatment with an aqueous

$30 \mathrm{NaOH}$ solution with 0 to 0.20 fraction concentrations. At $\mathrm{NaOH}$ concentrations less than 0.10 ,

31 the swelling occurred only along the tangential axis ( $\mathrm{T}$ ) and not along the radial (R) or

32 longitudinal (L) axes. At greater $\mathrm{NaOH}$ levels, the swelling was even more pronounced along $\mathrm{T}$

33 with shrinkage along the other axes. These anisotropic changes along $\mathrm{R}$ and $\mathrm{L}$ were closely

34 related to the crystallinity of microfibrils in the wood cell wall and simulated with a cell structure

35 model. This exercise revealed microfibril contraction and matrix swelling in the wood cell wall

36 upon $\mathrm{NaOH}$ treatment. The observed anisotropy in cross section was caused by differences in

37 the microfibril angles (LR and LT) with the cell wall.

38

39

40 


\section{Introduction}

Wood samples treated with aqueous $\mathrm{NaOH}$ show characteristic dimensional change, especially interesting along the longitudinal axis. Stöchmann (1971a, 1971b) discussed this phenomenon in detail and thermodynamically characterized the contraction of microfibrils in the wood cell wall, though the contraction mechanism of microfibrils themselves was not clarified. This contraction along the longitudinal axis is also induced by other alkaline solutions, the degree of which increases as $\mathrm{NaOH}<\mathrm{KOH}<\mathrm{LiOH}$ at the same concentration (Nakano, 1988a, 1988b, 1989). Nakano et al. (2000) found from relaxation behavior and temperature dependence studies that this contraction was due to the entropy elastic force of cell wall microfibrils, and formulated a relationship between the sample length, the microfibril dimensions, and microfibril angle.

Treatment with aqueous alkaline solutions also yields changes along the radial and tangential axes, which are negative and positive, respectively (Stöckmann, 1971a, 1971b), while the sum of their changes remains nearly constant (Ishikura and Nakano, 2007). The mechanism and a quantitative relationship between these anisotropic changes have not yet been elucidated. In the present work, the relationship between changes along all three axes upon exposure of wood samples to aqueous $\mathrm{NaOH}$ was established from simulations of a cell wall model. The results reported herein provide information about not only the anisotropic swelling of wood but also about the structure of the wood cell wall.

\section{Material and Methods}

The validity of the cell wall model employed herein was examined by comparing experimental data reported by Ishikura and Nakano (2007) with data calculated in the present work. Preparation condition in their report is followed. Yezo spruce, Saghalin fir, and Japanese larch, all soft woods that exhibit small microfibril angles, were prepared. Samples with dimensions of $20(\mathrm{R}) \times 20(\mathrm{~T}) \times 0.5(\mathrm{~L}) \mathrm{mm}$, where $\mathrm{R}$, T, and $\mathrm{L}$ correspond to the radial, tangential, and longitudinal axes, were soaked in aqueous $\mathrm{NaOH}$ solution with 0 to 0.20 fraction concentrations after drying under vacuum at room temperature over $\mathrm{P}_{2} \mathrm{O}_{5}$ for four days. The 
71 samples were stored at room temperature for two days and then washed in distilled water for one

72 week. Changes along each axis were calculated from the observed differences between wet

73 dimensions after $\mathrm{NaOH}$ treatment and the same measurements after soaking in distilled water

74 without $\mathrm{NaOH}$ treatment. Their dimensions were measured by a slide calipers for $\mathrm{R}$ and $\mathrm{T}$ and a 75 micrometer for L.

The crystallinity of the wood samples were determined from X-ray diffraction data obtained on the LR-plane at $30 \mathrm{kV}, 100 \mathrm{~mA}$, and $2 \%$ min. The degree of crystallinity was calculated from an the diffraction profile in the scanning range $10-32^{\circ}$. The profile was isolated to two parts of a non-crystalline and a crystalline, after subtracting air-scattering. The degree of crystallinity was defined as fraction of the crystalline reflection area to the gross area. As for the same samples, microfibril angles were freshly in this work measured by X-ray irradiation to know the effect of $\mathrm{NaOH}$ treatment on microfibril angles. In order to have higher precision LR-plane of a sample was measured at $40 \mathrm{kV}$ and $30 \mathrm{~mA}$, and then were calculated according to

84 Cave's method.

Results and discussion

Characterization of the dimensional change

Figure 1 represents experimental results, in which $\mathrm{NaOH}$ treatment induces dimensional changes not only along the longitudinal axis but also along the radial and tangential axes with $\Delta R / R<\Delta T / T$ in the cross section. At $\mathrm{NaOH}$ concentrations, [NaOH], greater than 0.10 fraction concentration, $\Delta R / R$ and $\Delta T / T$ values were less than and greater than zero,

94 respectively. It is clearly found that this anisotropically dimensional change differs from that 95 induced under water adsorption.

A suitable cell model is necessary to discuss the characteristic dimensional changes

97 shown in Fig. 1. Various cell models have been proposed to study features such as the anisotropic 98 swelling and shrinkage of wood and wood cells, including models proposed by Barber and Meylan 99 (1967), Yamamoto (1999), and Neagu and Gamstedt (2007), and the simplified model by Fratzl 100 (2008). Salmèn and Burgert (2009) reviewed recent reports describing cell -wall models. The 
101 modern cell model considers anisotropic microfibrils embedded in an elastic isotropic matrix; the 102 constitutive equation is derived under the equilibrium condition with adsorbed water in which 103 the matrix is isotropically swelling and microfibrils restrict this swelling. The microfiber angle is 104 an important factor for anisotropic dimensional change in the longitudinal direction and 105 perpendicular to the longitudinal direction. That is, the model is cylindrical. On the other hand, Preston (1942) and Sadoh and Kingston (1967) examined anisotropic swelling considering the geometrical deformation due to a swelling factor and some restraint.

Analysis of dimensional change should consider the interaction between microfibrils and the matrix for the anisotropy of a cross-section. A model of equilibrium between the matrix and microfibril restriction appears not to be suitable for simulation of anisotropic dimensional change because there is insufficient information on microfibril movement.

We follow the approach of Sadoh and Kingston (1967) in the discussion below. Dimensional change of the matrix and the microfibrils resulting from both the swelling and restraint induced by $\mathrm{NaOH}$ treatment is examined to analyze anisotropic dimensional change. Dimensional change in each direction is merely the sum of both contributions, assuming that the matrix swelling is restricted by an unknown factor which is evaluated by swelling weight, that each dimensional change is independent, and that change in the cell wall thickness is negligible The above assumptions imply that each dimensional change is described by the geometrical sum of the dimensional change of the microfibrils and that of the matrix with swelling weight.

Our model of anisotropic dimensional change also considers anisotropic microfibrils embedded in an elastic isotropic matrix. Note that microfibrils are contractible and that the microfibril angle is constant with $\mathrm{NaOH}$ treatment, as discussed below. wood treated with aqueous $\mathrm{NaOH}$ solution, assuming that distortion of the sample along the longitudinal axis depended on both microfibril contraction and a change in the microfibril angle.

$$
\frac{\Delta L}{L}=\frac{\Delta l}{l}+\frac{\Delta \cos \theta}{\cos \theta}
$$

where $L$ and $l$ are the sample length along the longitudinal axis and the microfibril length, respectively, $\cos \theta$ is the cosine of the microfibril angle $\theta$, and $\Delta L / L, \Delta l / l$ and $\Delta \cos \theta / \cos \theta$ represent the relative change in each of these variables. This equation is essentially the same as 
130 that of microfibrils derived by Sadoh and Kingston (1967) to discuss longitudinal shrinkage of

131 wood. It should be noted that the longitudinal axis of microfibril differs from that of the sample.

Lindström et al. (1988) examined the dependence of swelling behavior on $[\mathrm{NaOH}]$ for

milled wood lignin (MWL), which is the main matrix of wood gelled by epichlorohydrin. They evaluated the swelling of MWL not by dimensional changes but by weight gain. In their report, the water gain $(\mathrm{w} / \mathrm{w})$ was maximal at $0.8<[\mathrm{NaOH}]<1.6 \mathrm{meq} / \mathrm{g}$. The amount of weight gain increased gradually with increasing $[\mathrm{NaOH}]$ and increased rapidly at $[\mathrm{NaOH}]>20$ meq/g. This demonstrated that at high $\mathrm{NaOH}$ levels, lignin swelling was dependant on $\mathrm{NaOH}$ concentration. reduced to

$$
\frac{\Delta L}{L}=\frac{\Delta l}{l}+\frac{\Delta \cos \theta}{\cos \theta}+K_{1} \frac{\Delta m}{m}
$$

where $\Delta \mathrm{m} / \mathrm{m}$ is the swelling ratio of the matrix and $K_{1}$ is the swelling weight of matrix along the longitudinal axis of the sample. Restraint of matrix swelling is evaluated by the parameter $K_{1}$, which is probably caused by microfibrils. Figure 2 shows that the microfibril angle for Yezo spruce was on average $8.7^{\circ}$ and exhibited no dependence on $[\mathrm{NaOH}]$. The result shown in Figure 2 is different from the result by Fujimoto and Nakano (2000) that the fibril angle depended on $[\mathrm{NaOH}]$ using the iodine method. However, the results measured by X-ray irradiation was adopted in this work. This is because the X-ray diffraction method gives the average value of the fibril angles, though the iodine method does the local information for some cell walls. For wood species with very small microfibril angles, such as those used in the current study, where $\Delta \cos \theta=\Delta \theta \cdot \sin \theta \approx 0$, no dependence on [NaOH] was observed. The above equation then reduces to

$$
\frac{\Delta L}{L}=\frac{\Delta l}{l}+K_{1} \frac{\Delta m}{m}
$$

According to Equation (1), $K_{1} \Delta m / m \approx 0$ was expected since the contribution of the wood matrix to the change along the longitudinal direction was much smaller than that of microfibrils 155 because of the high elastic modulus of the microfibrils. However, dimensional changes in wood samples occur not only along the longitudinal axis but also along the radial and tangential axes

157 (Stöckmann, 1971a, 1971b; Ishikura and Nakano, 2007). Due to the small microfibril angle, 
158

159

160

161

162

163

164

165

166

167

168

170 along the radial and tangential axes of the sample are represented by

$$
\frac{\Delta R}{R}=\phi \frac{\Delta l}{l} \sin \theta_{L R}+K_{2}(1-\phi) \frac{\Delta m}{m},
$$

$$
\frac{\Delta T}{T}=\phi \frac{\Delta l}{l} \sin \theta_{L T}+K_{3}(1-\phi) \frac{\Delta m}{m},
$$
ratio is generally 0.3 or less for solid materials.

changes along the latter axes need to be considered in terms of matrix contributions. That is strikingly different from the dimensional change along the longitudinal axis. We will deal with not the mechanical equilibrium between wood components but the dimensional change resulted from both the swelling and restraint induced with $\mathrm{NaOH}$ treatment, as discussed below.

Expressions to describe the changes along the radial and tangential axes were initially derived assuming that the wood cell wall consists of two phases: the cellulose microfibril and an isotropic matrix, with a volume fraction microfibril / matrix $=\varphi /(1-\varphi)$, and microfibril angles of the LT- and LR-planes in wood cell wall designated as $\theta_{\mathrm{LT}}$ and $\theta_{\mathrm{LR}}$, respectively. Given this model structure, shown schematically in Figure 3, with a small microfibril angle, the changes

where the microfibril change along the longitudinal axis of itself is given by $\Delta l / l$, and dimensional changes along the radial and tangential axes of the sample are given by $\Delta R / R$ and $\Delta T / T$, respectively. Matrix contributions are represented by $\Delta m / m . K_{2}$ and $K_{3}$ are the swelling weight and are related to the anisotropy restraint of the radial and tangential orientations, respectively. or the Poisson ratio effect. If the Poisson ratio effect is different between LT-plane and LR-plane of wood cell due to the longitudinal shrinking as well as that for water sorption (Skaar, 1988), $\Delta T / T$ may be different from $\Delta R / R$. Considering that the microfibril angle is sufficiently small and that $\Delta R / R<\Delta T / T$ over $[\mathrm{NaOH}]$ as shown in Fig. $1,0<$ $K_{2}<K_{3}<1$. Dimensional change perpendicular to the longitudinal axis of the microfibrils is neglected in equations (2) and (3) because the contribution of $\Delta l / l$ is greater than that of the change perpendicular to the longitudinal axis of microfibrils. The reason is that the Poisson's 
The following discussion will focus on the implications of these dimensional changes as shown in Figure 1. At $[\mathrm{NaOH}]>0.10$, the signs in Equations (2) and (3) follow from Figure 1:

$$
\frac{\Delta R}{R}=\phi \frac{\Delta l}{l} \sin \theta_{L R}+K_{2}(1-\phi) \frac{\Delta m}{m}<0
$$

$$
\frac{\Delta T}{T}=\phi \frac{\Delta l}{l} \sin \theta_{L T}+K_{3}(1-\phi) \frac{\Delta m}{m}>0
$$

190 The sum of multiplying Equation (4) by $K_{3}$ and multiplying Equation (5) by $-K_{2}$ yields

$$
\phi \frac{\Delta l}{l}\left(K_{3} \sin \theta_{L R}-K_{2} \sin \theta_{L T}\right)<0,
$$

Considering that $0<\varphi<1,0<K_{2}<K_{3}<1$ and $0<\theta_{\mathrm{LT}}<\theta_{\mathrm{LR}}<90^{\circ}$ so that $\left(K_{3} \sin \theta_{L R}-K_{2} \sin \theta_{L T}\right)>0$ in Equation (6), the following expression is derived:

$$
\frac{\Delta l}{l}<0
$$

The experimental results of $\Delta R / R<0$ and $\Delta T / T>0$ in Figure 1 require $\Delta l / l<0$, that is, shows that the microfibril contracts along its longitudinal axis with $\mathrm{NaOH}$ treatment when Equations (1) to (3) are hold. The same result is also obtained from equation (1) because of $\Delta l / l \approx \Delta L / L<0$ due to $K_{1} \Delta m / m \approx 0$.

The above discussion suggests that microfibril contraction is the primary factor of anisotropic changes in wood. These changes can also be correlated with observed changes in the crystallinity of the microfibril at $\mathrm{NaOH}$ concentrations greater than 0.10 (Fengel et al., 1995). Figure 4 shows the relationship between crystallinity and dimensional changes along the longitudinal axis of samples. Changes were calculated based on the corresponding wet dimension at $[\mathrm{NaOH}]=0$. Contraction of the wood sample increased with decreasing crystallinity starting at $[\mathrm{NaOH}]=0.10$. The change shown in Figure 4 implies that a decrease in crystallinity causes contraction of the microfibril itself along the longitudinal axis, and hence an overall contraction of the sample along the longitudinal axis.

Two hypotheses can be considered for the location of the amorphous region created in the 
212 microfibril by $\mathrm{NaOH}$ treatment. One hypothesis is that the amorphous region exists about the 213 crystalline region as a square of side a few $\mathrm{nm}$ and expands with the cross section. The other 214 hypothesis is that the amorphous region forms at defects along the longitudinal axis of the 215 microfibril which exist by about $20 \mathrm{~nm}$ and extends with a length along the longitudinal axis. 216 The 20-nm length has been confirmed by leveling-off degree of polymerization (LODP) 217 experiments and X-ray diffraction (Hayashi et al., 1989). The high linear correlation of $\Delta L / L$ 218 as a function of crystallinity shown in Figure 4 suggests that $\mathrm{NaOH}$ treatment induces 219 crystallinity changes in one of the aforementioned regions. Nakano et al. (2000) reported that 220 this change in crystallinity is localized in the longitudinal region of microfibril rather than the cross section.

Figure 5 shows the proposed combination mode of crystallinity of microfibers after $\mathrm{NaOH}$ treatment, where the crystallinity along the longitudinal and cross-sectional axes are $w_{c}$ and $h_{c}$, respectively. That is, they are the contribution of parallel and series to the longitudinal axis of microfibrils. The gross crystallinity, $\underline{\zeta}$, is represented by

This equation should not be a linear function of $[\mathrm{NaOH}]$ if the both crystallinity $w_{c}$ and $h_{c}$ have a $[\mathrm{NaOH}]$ dependence. However, $\zeta$ as a function of $\mathrm{NaOH}$ concentration shows a highly correlated linear relationship, as shown in Figure 6. The crystallinity of wood samples $\zeta$ is approximately used as that of microfibrils in wood. This provides additional evidence that the crystallinity changes causes in either region of the both. Therefore, it can be concluded that exposure to aqueous $\mathrm{NaOH}$ induces changes in crystallinity along the longitudinal axis, which in turn induces microfibril contraction.

he crystallinity of the wood samples were determined from X-ray diffraction data obtained on the LR-plane at $30 \mathrm{kV}, 100 \mathrm{~mA}$, and $2 \% \mathrm{~min}$. The degree of crystallinity was calculated from an the diffraction profile in the scanning range $10-32^{\circ}$. 

for $\Delta R / R$ versus $\Delta L / L$ and $\Delta T / T$ versus $\Delta L / L$, where $K_{1} \Delta m / m$ is neglect because of $K_{1} \Delta m / m=0.0010$ :

$$
\frac{\Delta R}{R}=\phi \frac{\Delta L}{L} \sin \theta_{L R}+K_{2}(1-\phi) \frac{\Delta m}{m}
$$

$$
\frac{\Delta T}{T}=\phi \frac{\Delta L}{L} \sin \theta_{L T}+K_{3}(1-\phi) \frac{\Delta m}{m}
$$

250 Microfibril angles in the LR- and LT-planes can be calculated using the first derivatives of 251 Equations (9) and (10) as a function of $\Delta L / L$, which are confirmed by experimental results at $252[\mathrm{NaOH}]>0.10$ for $\Delta R / R$ versus $\Delta L / L$ and $\Delta T / T$ versus $\Delta L / L$. The second terms in both equations are regarded as constant, since $\Delta R / R$ and $\Delta T / T$ are almost constant and $\Delta L / L=0$ at $[\mathrm{NaOH}]<0.10$.

Experimental results yielded slope values of 0.0800 for $\Delta R / R$ versus $\Delta L / L$ and 0.0188 for $\Delta T / T$ versus $\Delta L / L$, with $y$ intercepts of 0.0045 and 0.0305 , respectively. Accordingly, microfibril angles of $\theta_{\mathrm{LR}}=9.20^{\circ}$ and $\theta_{\mathrm{LT}}=2.15^{\circ}$ were obtained. The former value agrees reasonably well with the average microfibril angle observed in the LR-plane shown in Figure 2, though the latter appears to be too little comparing the results reported (Nakato, 1958) In the present report, these values were deemed valid and used in the below simulation.

\section{Simulation of the anisotropic dimensional change}

The main parameter affecting dimensional changes in the wood samples was crystallinity changes as a result of the $\mathrm{NaOH}$ treatment shown in Figure $4 . \mathrm{NaOH}$ induces a longitudinal axis and ultimately a contraction of the wood sample along the longitudinal axis of the sample. It should be noted that the longitudinal axis of microfibril differs from that of the sample. Although this effect is manifest primarily along the longitudinal axis of the sample, the 
270

271

272

273

274

275

276

277

278

279

280

281

282

283

284

285

286

287

288

289

290

291

292

293

294

295

296

297

298

tangential and radial regions are also considered in the below simulation.

From Equation (1), the dimensional change of the sample, $\Delta L / L$, with the experimentally derived $K_{1} \Delta m / m=0.0010$, can be represented by

$$
\frac{\Delta L}{L}=\frac{\Delta l}{l}+0.0010
$$

where the value 0.0010 represents the contribution of matrix swelling to the overall dimensional change of the sample along the longitudinal axis at $[\mathrm{NaOH}]=0.03$ to 0.07 and is negligible small. The value $\Delta L / L$ was calculated from the contraction of the microfibril, $\Delta l / l$, as shown in Equation (11). Thus, determination of $\Delta L / L$ can be reduced to $\Delta l / l$, which in turn can be evaluated from the end-to-end distance of the amorphous cellulose chain along the longitudinal axis of the microfibril.

It is difficult to know the actual behavior of the cellulose chain when both ends may be bound to a crystalline region, though some researchers reported the simulation of conformation of single cellulose chain in this regard (for example Queyroy et al., 2004). Additionally, the end-to-end distance of the cellulose chain appears to be slightly influenced by non-crystallization because of a non-flexible polymer chain. Therefore, the classical method by Benoit (1947), using the characteristic parameter a to represent a corrected deviation from the free jointed chain model, was utilized herein.

As well-known, the end-to-end distance of the cellulose chain is represented by $0.79 \sqrt{N}$ for the unit number $N$ when the appropriate constants are substituted in Benoit's equation: a $\mathrm{C}-$ $\mathrm{O}$ bond length of $0.143 \mathrm{~nm}$, a length between $\mathrm{C}_{1}$ and $\mathrm{C}_{4}$ equal to $0.298 \mathrm{~nm}$, and angles $\mathrm{COC}$ and $\mathrm{C}_{1} \mathrm{C}_{4} \mathrm{O}$ of $110^{\circ}$ and $150^{\circ}$, respectively. Benoit's equation requires the correction term $\alpha$, representing the length of the free jointed chain model. For example, a is typically 2-5 for cellulose tributylate and cellulose tricaplate and about 2 for polyethylene. The former values are representative of a rod-like polymer conformation and the latter has a simple, flexible structure. Thus, the length of the amorphous region along the longitudinal axis of microfibril $l_{a}$ is described by $l_{a}=0.79 \alpha \sqrt{N}$.

The change in microfibril length, $\Delta l / l$, can be represented by the degrees of non-crystallization of microfibrils with $\mathrm{NaOH}$ treatment, with microfibril length, the length of a glucose residue, and crystallinity along the longitudinal axis given by $l_{u}, l_{g}$, and $h_{c}\left(=\zeta / w_{c}\right)$, 
respectively:

300

$$
\frac{\Delta l}{l}=\left(l_{u} \cdot h_{c}+0.79 \alpha \sqrt{\frac{l_{u}\left(1-h_{c}\right)}{l_{g}}}\right) / l_{u}-1
$$

301

302

303

304

305

306

The first and second terms in parentheses of Equation (12) are the length of the crystalline and amorphous regions, respectively. The first term in Equation (12) is the ratio of microfibril length after / before $\mathrm{NaOH}$ treatment. The microfibril length $l_{u}$ is the period determined from LODP and X-ray diffraction with 20nm. Substituting $l_{u}=20 \mathrm{~nm}$ and $l_{g}=0.515 \mathrm{~nm}$ into Equation (12) and then substituting this result into Equation (11) yields

$$
\frac{\Delta L}{L}=\left[\left(20 \cdot h_{c}+0.79 \alpha \sqrt{\frac{l_{u}\left(1-h_{c}\right)}{0.515}}\right) / 20-1\right] \cos \theta+0.0010
$$

Dimensional changes in the wood samples as a function of crystallinity induced by $\mathrm{NaOH}$ treatment can now be simulated using Equations (8) and (13). The simulation variables were $h_{c}\left(=\zeta / w_{c}\right)$ and $\alpha$, since the empirical microfibril angles were small $\left(\theta_{\mathrm{LR}}=9.20^{\circ}\right.$ and $\theta_{\mathrm{LT}}=$ $2.15^{\circ}$ so that $\cos \theta_{L R} \approx \cos \theta_{L R} \approx 1$ ). Typical results in Figure 7 show that the most accurate simulations were obtained with $\alpha=3$, when $w_{c}=1.00$ and then $h_{c}\left(=\zeta / w_{c}\right)=0.522$ where $\zeta$ the gross crystallinity. This implies that changes in crystallinity occurred only along the longitudinal axis of microfibrils and that that the amorphous cellulose chains are extended.

Table 1 shows the results obtained with $\alpha=2.5$ and 3 . The degree of contraction calculated from the ratio of end-to-end distance to contour length was about 0.10-0.20. According to analyses of single cellulose chains by Queyroy et al. (2007), the end-to-end distance of a chain consisting of eight glucose residues with a contour length of $4.4 \mathrm{~nm}$ is about $3.2 \mathrm{~nm}$. This corresponds to a 0.30 contraction. Considering $N=13$ to 26 in this work and $N=8$ in Queyroy's simulation, the former is much smaller than the latter. This may simply result from the different calculation methods employed. However, the most likely reason is that both ends of the amorphous cellulose chains bond to the crystalline regions and are therefore unable to satisfactorily contract. This conclusion is also supported by the simulation results obtained with $\alpha=3$.

The observed changes in the radial and tangential directions, $\Delta R / R$ and $\Delta T / T$, were also simulated based on derivations from Equations (9) and (10). Simulation requires the parameters $K_{1}$ and $K_{2}$. Their contribution can be estmated using $\Delta R / R$ and $\Delta T / T$ at $[\mathrm{NaOH}]$ 
$327<0.10$ since the microfibril does not contract at $[\mathrm{NaOH}]<0.10$, where dimensional changes are 328 due to changes in the matrix only:

$$
\frac{\Delta R}{R}=K_{2}(1-\phi) \frac{\Delta m}{m}
$$

$$
\frac{\Delta T}{T}=K_{3}(1-\phi) \frac{\Delta m}{m}
$$

331 Both $\Delta T / T$ and $\Delta R / R$ varied little at $[\mathrm{NaOH}]<0.1$ and were regarded as constant Empirical results were $\Delta R / R=K_{2}(1-\phi) \Delta m / m=-0.0037$ and $\Delta T / T=K_{3}(1-\phi) \Delta m / m=0.0217$. Similarly, $333 K_{1} \Delta m / m=0.0010$ was obtained from empirical result at $[\mathrm{NaOH}]<0.1$ and Equations (1). That is, $K_{1}$ and $K_{2}$ are almost 0 and $K_{3}>0$ at $[\mathrm{NaOH}]<0.1$, considering swelling of matrix. This fact suggests strong restraint along the longitudinal and radial axes and little restraint along tangential axis.

As shown in Fig.1, $\Delta T / T$ was constant swelling and $\Delta R / R$ varied little at $[\mathrm{NaOH}]$ between 0.03 and 0.10 where no longitudinal shrinking. This fact suggests that wood matrix is only slightly dependent on $\mathrm{NaOH}$ concentration though it swells due to the $\mathrm{NaOH}$ treatment.

340 Thus, assuming that dimensional changes in the wood matrix were constant over $[\mathrm{NaOH}]=0.03$ to 0.20, $\Delta T / T$ and $\Delta R / R$ were calculated using Equations (2) and (3). The most accurate results for $\Delta L / L$ were obtained with $\alpha=3, h_{c}=0.522, w_{c}=1.00, \theta_{\mathrm{LT}}=2.15^{\circ}$, and $\theta_{\mathrm{LR}}=9.20^{\circ}$. These results are shown in Figure 1 as solids lines which are best fitting and show good agreement between experimental and calculation data. Some disagreement, however, can be observed along the tangential axis and may be due to the Poisson ratio effect, which was not accounted for in these simulations. Low microfibril angles allow the matrix to swell easily along the in tangential axis. Additional changes due to the Poisson effect may then be caused by shrinking

348 along the radial axes. This effect may also contribute to the observed discrepancy between experimental and theoretical results in Figure 1 at $[\mathrm{NaOH}]>0.10$.

\section{Conclusion} to 0.20 fraction concentrations was examined. At $\mathrm{NaOH}$ concentrations less than 0.10 , swelling 
355 occurred only along the tangential axis $(\mathrm{T})$ and not along the radial (R) or longitudinal (L) axes. 356 At greater $\mathrm{NaOH}$ levels, the swelling was even more pronounced along $\mathrm{T}$, with shrinkage along 357 the other axes. These anisotropic changes along $\mathrm{R}$ and $\mathrm{L}$ were closely related to the longitudinal 358 crystallinity change of microfibrils in the wood cell wall and were simulated with the cell 359 structure model. This exercise revealed microfibril contraction and matrix swelling in the wood 360 cell wall upon $\mathrm{NaOH}$ treatment. That is, the characteristic dimensional changes in wood with $361 \mathrm{NaOH}$ treatment are due to the decreasing crystallinity of microfibrils, matrix swelling, and 362 differences in microfibril angles in the LP= and LT= planes of the wood cell wall. The main factor 363 is microfibril contraction along the longitudinal axis with decreasing crystallinity in the 364 microfibrils. The results obtained in this analysis illustrate the complex interaction between 365 microfibrils and the matrix and may serve as a new basis for understanding the anisotropic 366 swelling and shrinkage of wood.

Acknowledgements 
374

375

376

377

378

379

380

381

382

383

384

385

386

387

388

389

390

391

392

393

394

395

396

\section{References}

Barber NF, Meylan BA (1964) The anisotropic shrinkage of wood. Holzforschung 18: 146-156

Benoit H (1947) Sur la statique des chains avec interactions et empechements ateriques. J Chem Phys 44: 18-21

Fengel D, Jakob H, Strobel C (1995) Influence of the alkali concentration on the formation of cellulose II. Holzforschung 49: 505-511

Fratzl P, Elbaum R, Burgert I (2008) Cellulose fibrils direct plant organ movement. Faraday Discussion 139: 275-282

Hayashi J, Ishizu A., Isogai A (1989) Chemistry and solid state structure of cellulose. Sen-i Gakkaishi 45: 469-480

Ishikura Y., Nakano T (2007) Contraction of the microfibrils of wood treated with aqueous $\mathrm{NaOH}$. J Wood Sci 53: 175-177

Lindström T, Wallis A, Tulonen J, Kolseth P (1988) The effect of chemical environment on swelling and dynamic mechanical properties of milled wood lignin gels. Holzforschung 42 : $225-228$

Nakato K. (1958) On the cause of the anisotropic shrinkage and swelling of wood IX (in Japanese). Mokuzai Gakkaishi 4: 134-141

Nakano T (1988a) Plasticization of wood by alkali treatment (in Japanese). Nihon Reoroji Gakkaishi (Journal of the society of Rheology, Japan) 16(1): 48-49

Nakano T (1988b) Plasticization of wood by alkali treatment effects of kind of alkali and concentration of alkaline aqueous solution on stress relaxation (in Japanese). Nihon Reoroji Gakkaishi (Journal of the society of Rheology, Japan) 16(3): 104-110

Nakano T (1989) Plasticization of wood by alkali treatment. Relationship between plasticization and the ultra-structure (in Japanese). Mokuzai Gakkaishi 35(5): 431-437

Nakano T, Sugiyama J, Norimoto M (2000) Contraction force and transformation of microfibril with aqueous sodium hydroxide solution. Holzforschung 54: 315-320

Preston, R.D. 1942. Anisotropic contraction of wood and its constituent cells. Forestry, 16, $32-48$.

Queyroy S, Müller-Plathe F, Brown D (2004) Molecular dynamics simulations of cellulose oligomers conformational analysis. Macromol Theory Simul 13: 427-440 
404 Sadoh T, Kingston RST (1967) Te relation between longitudinal shrinkage and structure. Wood \& 405 Technol. 1:81-98

406 Salmén L, Burgert I (2009) Cellwall features with regard to mechanical performance. $407 \quad$ Holzforschung 63: 121-129

408 Skaar, C. (1988) Wood-Water Relations. Springer, Berlin Heidelberg New York London Paris 409 Tokyo, pp 155-156

410 Stöckmann VE (1971a) Effect of pulping on cellulose structure Part I. Tappi 54(12): 2033-2037

411 Stöckmann VE (1971b) Effect of pulping on cellulose structure Part II. Tappi 54(12): 2038-2045

412 Fujimoto T, Nakano T (2000) The effect of mercerization on wood structural features. Mokuzai 413 Gakkaishi 46(3): 238-241

414 Yamamoto H, Kojima Y, Okuyama T, Abasolo WP (2002) Origin of the biomechanical properties of 415 wood related to the fine structure of the multi-layered cell wall. J Biomechanical Eng 124: $416 \quad 432-440$ 


\section{Figure captions}

Fig. 1. Experimental (plots) and best fitting (solid lines) results of dimensional changes along the radial ( ), tangential ( $\square$ ), and longitudinal (০) axes as functions of the $\mathrm{NaOH}$ aqueous solution. Note: $\mathrm{a}=3, \theta_{L T}=2.15^{\circ}$ and $\theta_{L R}=9.20^{\circ}$.

Fig. 2. Dependence of microfibril angle on the concentration of aqueous $\mathrm{NaOH}$ solution.

Fig. 3. The cell wall model for anisotropic dimensional changes consisted of matrix and microfibril components. Note: $\theta=$ fibril angle, $\varphi=$ volume fraction of the microfibril.

Fig. 4. Dimensional changes along the longitudinal axis of treated wood as functions of crystallinity.

Fig. 5. A schematic diagram for the crystalline and amorphous regions of a microfibril.

Note: $w_{c}$ and $h_{c}$ are crystallinity of micro fibril in cross section and longitudinal directions.

Fig. 6. Relationship between crystallinity and concentration of aqueous $\mathrm{NaOH}$ solution for Yezo spruce treated with aqueous $\mathrm{NaOH}$ solution.

Fig. 7. Typical experimental (open circle) and simulated (solid line) results of dimensional changes along the longitudinal axis of wood samples. Note: $\theta=$ fibril angle; $\alpha=$ characteristic ratio; $w_{c}=$ crystallinity of the cross section. 
Table 1. Typical end-to-end distances, representing the highest degree of agreement between experimental and theoretical results in amorphous regions of $20-\mathrm{nm}$ microfibrils.

Note: $w_{c}=$ crystallinity of the cross-sectional area; $h_{c}=$ crystallinity of the longitudinal area; $\mathrm{N}=$ number of glucose residues in the amorphous region; $\alpha=$ characteristic ratio.

\begin{tabular}{cccccccc}
\hline & \multicolumn{3}{c}{$w_{c}=1.00$} & \multicolumn{3}{c}{$w_{c}=0.70$} \\
Crystallinity $\zeta$ & $h_{c}$ & $N$ & $\begin{array}{c}\alpha=3 \\
\text { Distance }(\mathrm{nm})\end{array}$ & $h_{c}$ & $N$ & $\begin{array}{c}\alpha=2.5 \\
\text { Distance (nm) }\end{array}$ \\
\hline 0.459 & 0.459 & 21 & 10.9 & 0.656 & 13 & 7.2 \\
0.428 & 0.428 & 22 & 11.2 & 0.611 & 15 & 7.7 \\
0.380 & 0.380 & 24 & 11.6 & 0.543 & 18 & 8.3 \\
0.351 & 0.351 & 25 & 11.9 & 0.501 & 19 & 8.7 \\
0.320 & 0.320 & 26 & 12.2 & 0.457 & 21 & 9.1 \\
\hline
\end{tabular}




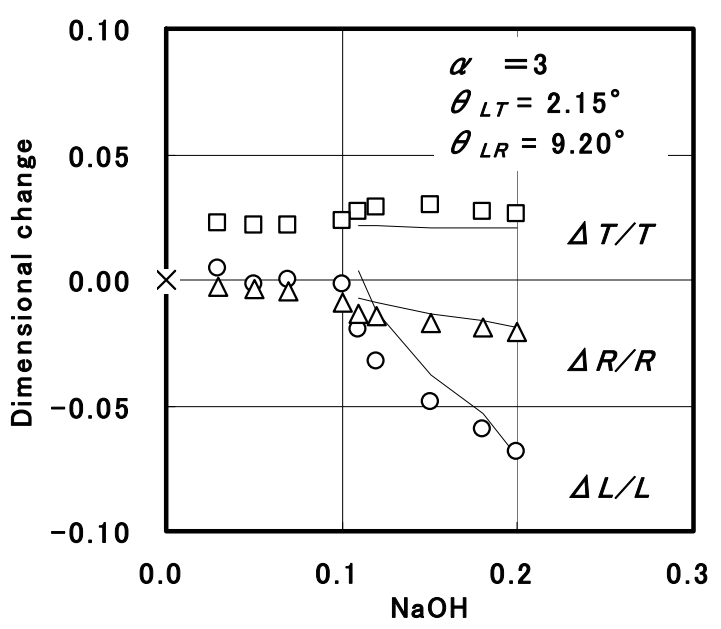

Fig. 1. Experimental (plots) and best fitting (solid lines) results of dimensional changes along the radial ( ), tangential ( $\square$ ), and longitudinal (০) axes as functions of the $\mathrm{NaOH}$ aqueous solution. Note: $\alpha$ (characteristic ratio of polymer conformation) $=3, \theta_{L T}=2.15^{\circ}$ and $\theta_{L R}=9.20^{\circ}$. 


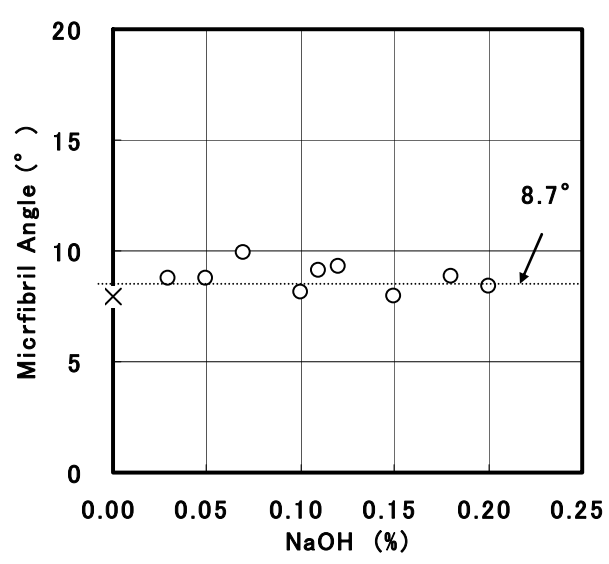

Fig. 2. Dependence of microfibril angle on the concentration of aqueous $\mathrm{NaOH}$ solution. 

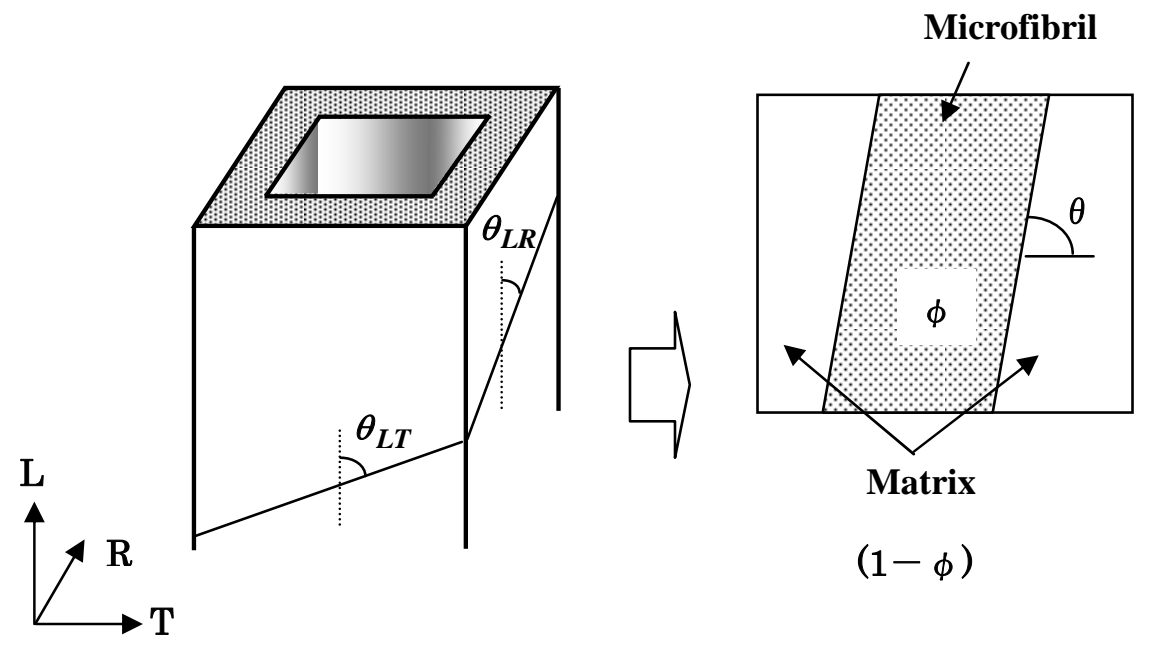

$$
(1-\phi)
$$

Fig. 3. The cell wall model for anisotropic dimensional changes consisted of matrix and microfibril components. Note: $\theta=$ fibril angle, $\varphi=$ volume fraction of the microfibril. 


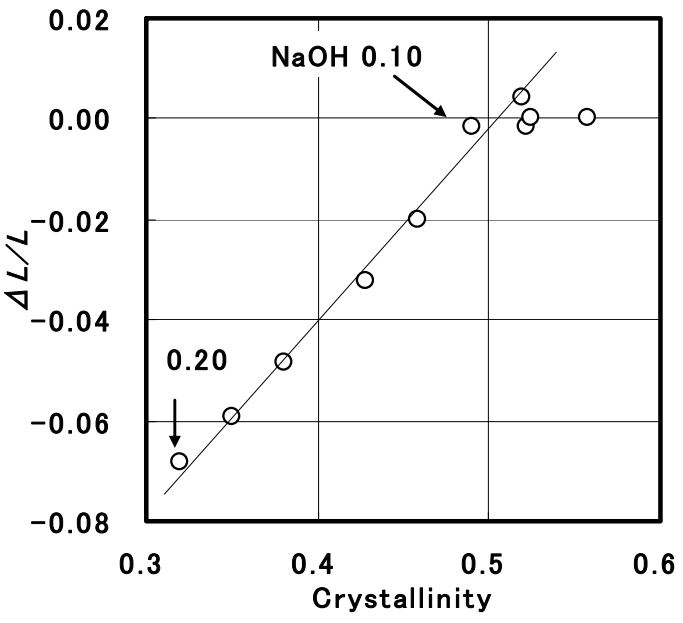

Fig. 4. Dimensional changes along the longitudinal axis of treated wood as functions of crystallinity. 


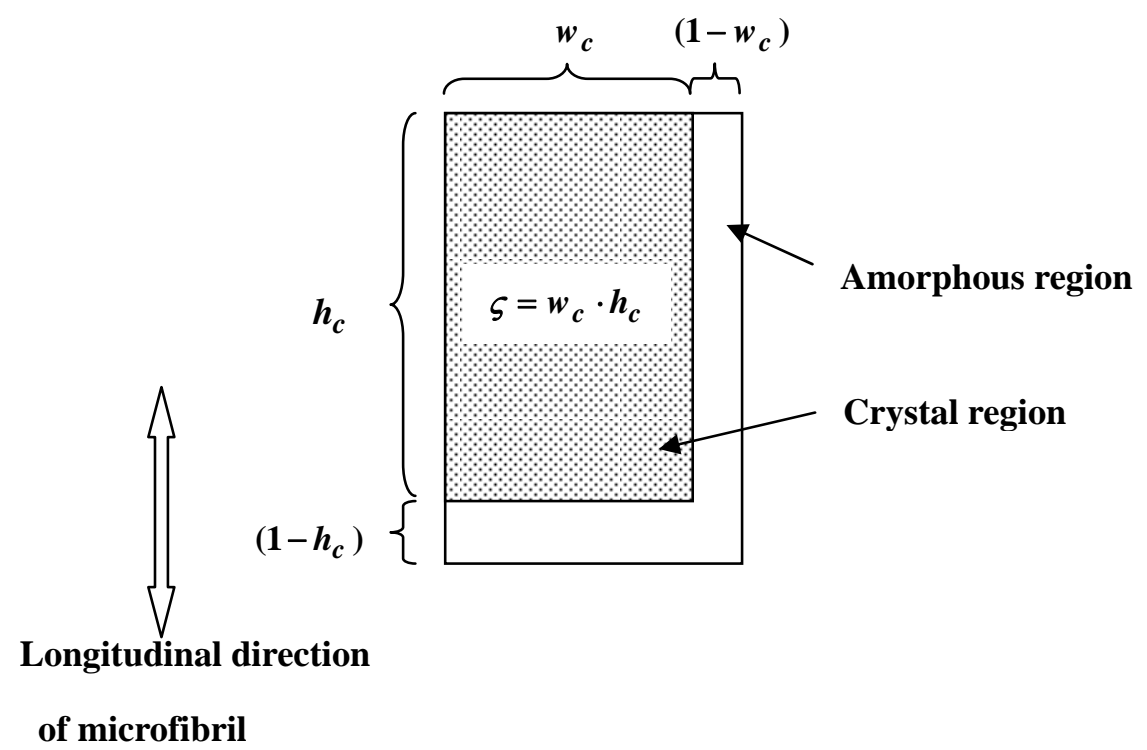

Fig. 5. A schematic diagram of combination mode for the crystalline and amorphous regions of a microfibril. Note: $w_{c}$ and $h_{c}$ are crystallinity of micro fibril in cross section and longitudinal directions. 


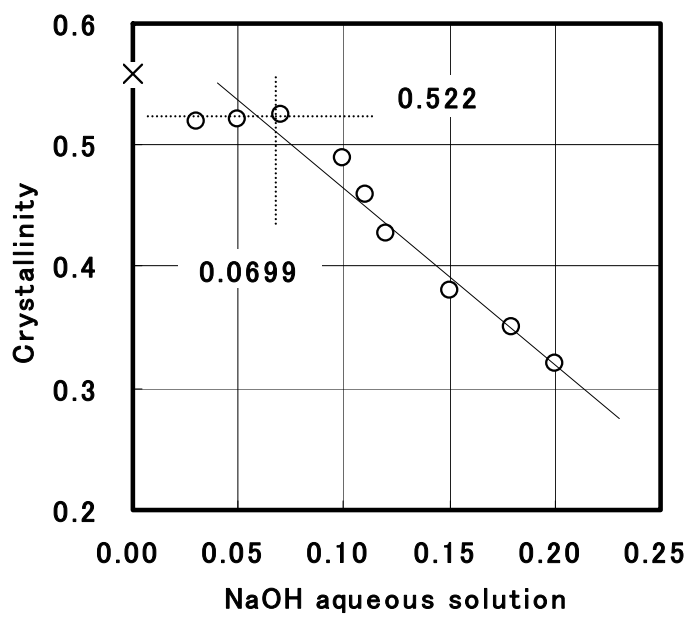

Fig. 6. Relationship between crystallinity and concentration of aqueous $\mathrm{NaOH}$ solution for Yezo spruce treated with aqueous $\mathrm{NaOH}$ solution. 

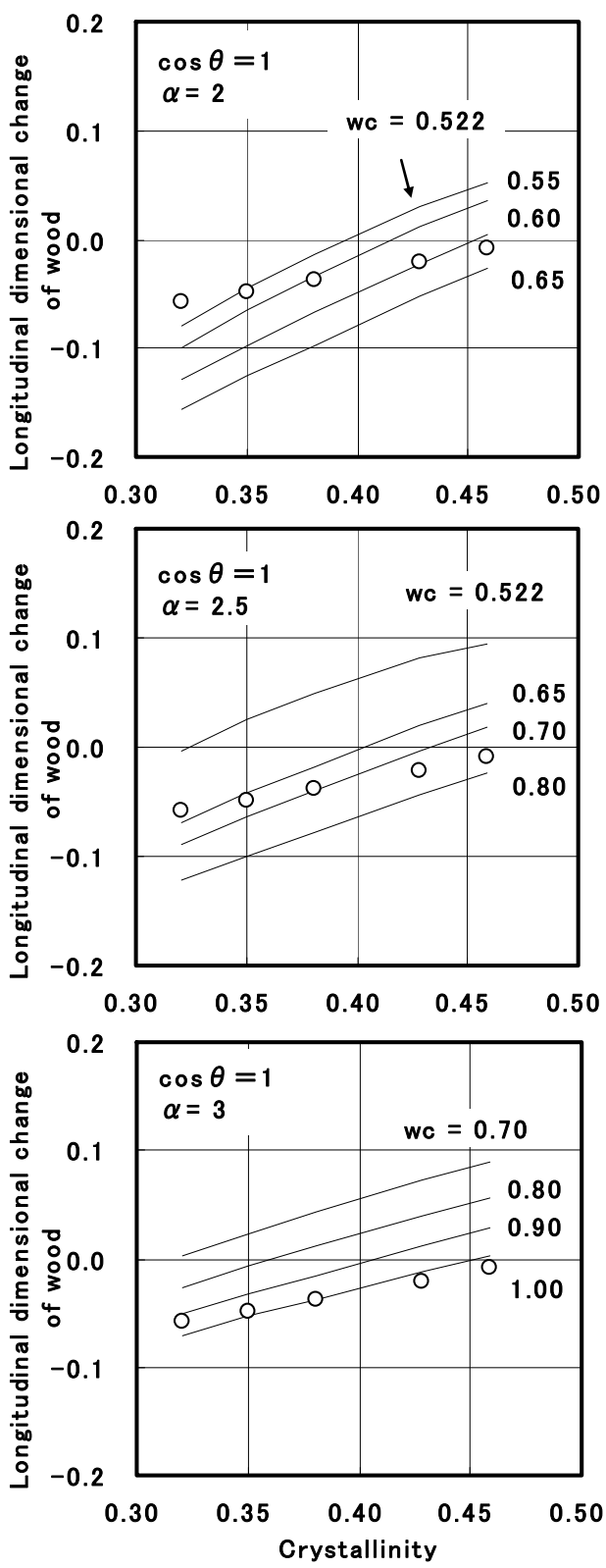

Fig. 7. Typical experimental (open circle) and simulated (solid line) results of dimensional changes along the longitudinal axis of wood samples. Note: $\theta=$ fibril angle; $\alpha=$ characteristic ratio; $w_{c}=$ crystallinity of the cross section. 